\title{
Performance evaluation of a MIMO channel model for simplified OTA test systems
}

\author{
Sahrul Pasisingi, Katsuhiro Nakada, Akira Kosako and Yoshio Karasawa*
}

\begin{abstract}
This paper shows performance evaluation results of the proposed multiple-input multiple-output (MIMO) channel model with simplified configuration for OTA measurement systems. The key feature of our proposal is the adoption of a simple antenna branch-controlled configuration for generating various Rayleigh fading environments composed of a number of multipath delayed waves. The scheme matches well with FPGA implementation with IF band signal processing. The channel model and its theoretical background, and the basic configuration of the measurement system are presented. From an eigenvalue analysis of the generated channel matrix, it is verified that an 8-probe antenna system can realize a very accurate multipath environment for the performance measurement of a $2 \times 2$ MIMO system, while a 12- or 16-probe antenna system can do the same for a $4 \times 4 \mathrm{MIMO}$ system.
\end{abstract}

Keywords: MIMO; OTA; Fading emulator; Wideband channel; Propagation channel model

\section{Introduction}

Over-the-air (OTA) test methods are of growing interest for the evaluation of multiple-input multiple-output (MIMO) terminals in LAN, WiMAX, LTE, and other wireless communication systems that adopt MIMO technologies. In OTA testing, a realistic propagation environment is generated around the receiving terminals for measurement of transmission performance characteristics. For OTA system development, the multipath propagation channel models that have been proposed thus far, such as [1-4], have been used in clarifying fundamental concepts. Standardization of OTA measurement methods is currently under consideration by the Third Generation Partnership Project (3GPP) [5].

In OTA test methods, the environment for MIMO terminal evaluation is generated by either a reverberation chamber or a fading emulator $[5,6]$. In the reverberation chamber system, a chamber with metallic walls that effectively reflect waves is used to generate a rich multipath propagation environment [5,7]. In fading emulator systems, hereinafter denoted as FE systems, a number of virtual scattering probe antennas are positioned circularly around the terminals being tested (devices under test, DUTs) in order to generate a fading environment [8-13]. Although both

\footnotetext{
* Correspondence: karasawa@ee.uec.ac.jp

The University of Electro-Communications, 1-5-1 Chofugaoka, Chofu, Tokyo 182-8585, Japan
}

methods have advantages and limitations, we investigated FE OTA systems with a focus on channel control flexibility. Among FE MIMO-OTA systems, time-varying multipath signal generation using an available fading simulator scheme is a promising candidate in 3GPP [5] because the fading simulator is able to flexibly control propagation parameters such as delay spread, Doppler spread, and angular spread. Although this type of system can easily realize all necessary functions, the construction cost is generally extremely high. Therefore, in our previous papers [14,15], we proposed an FE MIMO-OTA system with a simplified configuration named as the antenna branch-controlled system and carried out narrowband experiments without any delayed paths. In addition, field-programmable gate array (FPGA) implementation of the proposed scheme has been conducted [16]. Although performance identification of the proposed channel model is essential for system development, quantitative evaluations have not yet been achieved in previous papers.

Accordingly, in this paper, after introducing the channel model and its theoretical background, we will make clear the generated channel performance as a function of the number of probe antennas. From an eigenvalue analysis of the generated channel matrix, we verify that an 8-probe antenna system can realize a very accurate multipath environment for the performance measurement of a $2 \times 2$ MIMO system, while a 12- or 16-probe antenna system can do the same for a $4 \times 4$ MIMO system. 


\section{Antenna branch-controlled FE-type MIMO-OTA system and its channel model \\ 2.1 FE-type MIMO-OTA system}

Figure 1 shows the basic configuration of an FE-type MIMO-OTA measurement system. The main components of the system are the transmitting antenna input ports $(M$, in number), the probe antennas which realize the actual multipath environment $(L)$, the receiving antennas $(N)$ of the device (terminal) under test, and the $L \times M$ network for multipath channel generation. The $L \times M$ network, which connects the transmitting antenna ports and the probe antennas, plays an essential role in controlling the signals used to generate the desired propagation channels.

Figure 2 shows two schemes of the FE-type system for generating a multipath fading environment with the time-varying amplitude and phase of $M L$ paths. Figure 2a shows the configuration adopted in most available systems. We call this the 'path-controlled type' or 'FE-1' configuration. As stated above, the FE-1 configuration uses a number of fading simulator units that can flexibly control the amplitude, phase, and delay. Since the use of such high-performance fading simulator units with $M L K$ ( $K$, the number of delayed paths) Rayleigh faders is needed, the cost of system construction is high. Figure $2 \mathrm{~b}$, in contrast, is the simplified configuration $[14,15]$ evaluated in this paper. We denote this as the 'antenna branchcontrolled type' or 'FE-2' configuration. Even when a number of delayed waves are present, the necessary number of Doppler shifters in place of Rayleigh faders is $L$ only.

In the first stage, the desired spatial correlation characteristics between $M$ transmitting antenna ports and $L$ probe antennas are realized by a fixed matrix connection. The second stage generates multipath delays and individual Doppler shifts branch by branch. This kind of functional separation results in a very simple and practical configuration. Figure 3 shows a more detailed functional diagram of the proposed system [14]. The FE-2 configuration enables independent Rayleigh fading

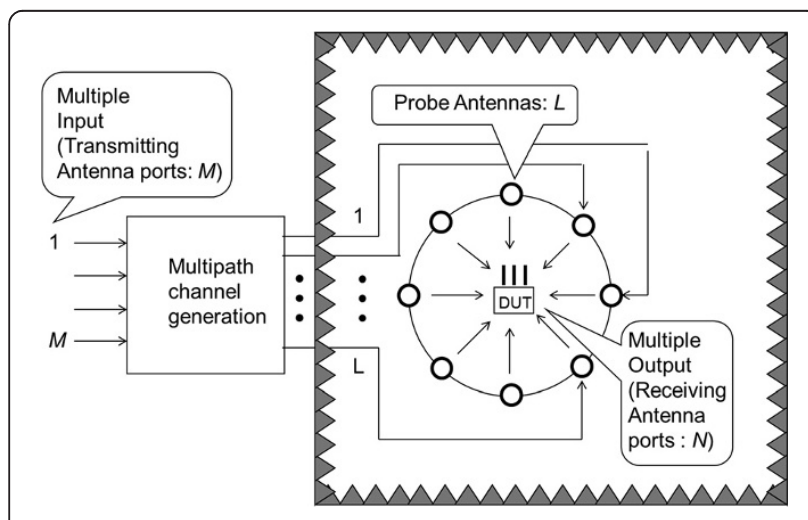

Figure 1 General configuration of an FE-type MIMO-OTA system.

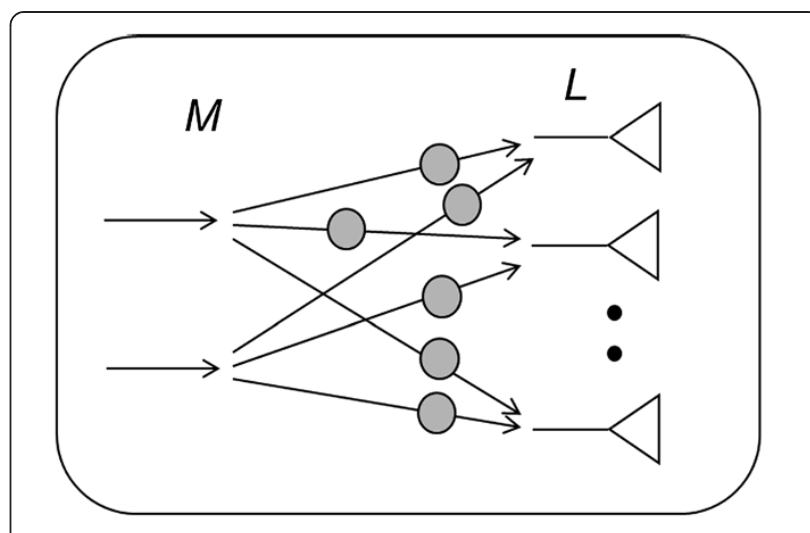

(a) Path-controlled type (FE-1)

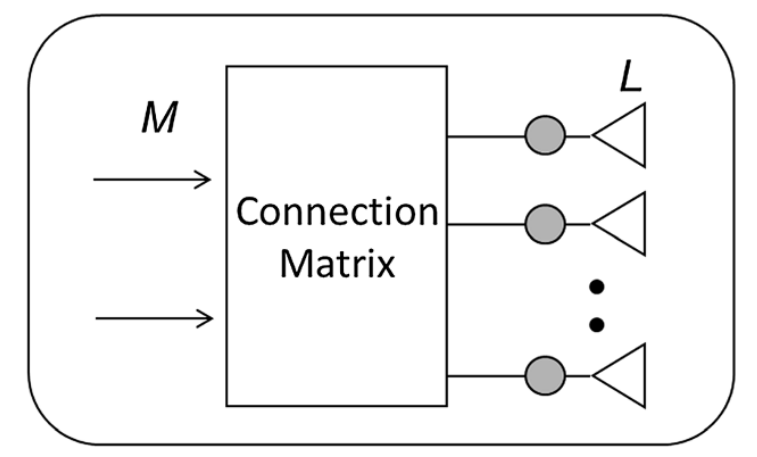

(b) Antenna-branch-controlled type (FE-2)

Figure 2 Functional configuration of 'multipath channel generation' shown in Figure 1. Gray circle, multipath generation function, namely Rayleigh fader for FE-1 and Doppler shifter for FE-2. (a) Path-controlled type (FE-1). (b) Antenna branch-controlled type (FE-2).

for different input signal ports when receiving those signals at a receiving point via a fixed matrix connection using Walsh-Hadamard (WH) codes [14,17]. By suitably adjusting each delayed path weight, the spatial combination of the signals from those antennas produces independent Rayleigh fading. This configuration, unlike FE-1, does not require the generation of any Rayleigh variations in the network. Since it does not include time-varying functions in the signal processing stage, its structure is quite simple and easily constructed.

\subsection{Channel model for the FE-2 system}

The MIMO channel model for the FE-2 system can be expressed after functional decomposition as follows [14]:

$$
\boldsymbol{H}(t, \tau)=\boldsymbol{A}_{\mathrm{RX}} \boldsymbol{A}_{\text {Doppler }}(t) \boldsymbol{H}_{\text {delay }}(\tau) \boldsymbol{W}_{\mathrm{TX}}
$$

where matrices $\boldsymbol{W}_{\mathrm{TX}}, \boldsymbol{H}_{\text {delay }}, \boldsymbol{A}_{\text {Doppler }}$ and $\boldsymbol{A}_{\mathrm{RX}}$ are the transmission connection matrix, the impulse response 


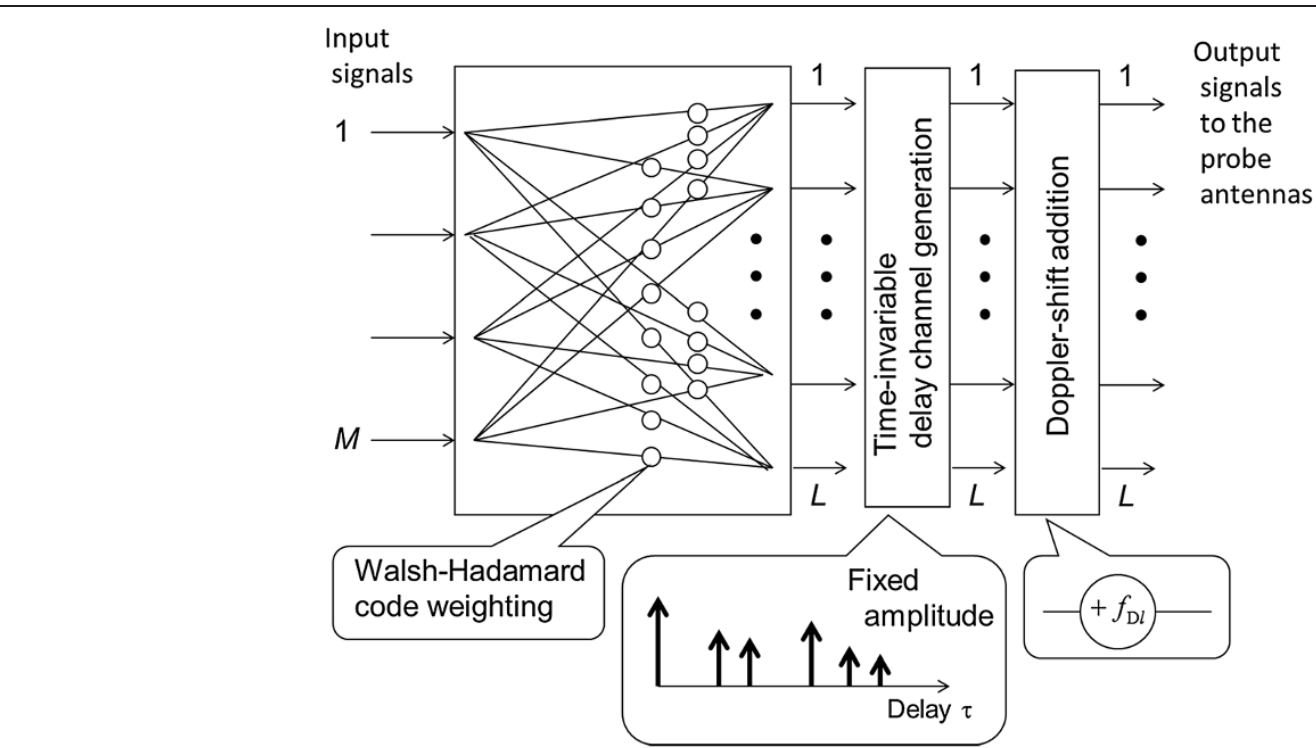

Figure 3 Detailed functional block diagram of the FE-2 system (proposed configuration).

matrix, the Doppler shift matrix, and the reception channel matrix, respectively. All the matrices in the equation are described in detail in [14] and are summarized in Figure 4. Finally, the overall channel impulse response can be obtained by

$$
\boldsymbol{H}(t, \tau)=\sum_{k=1}^{K} \boldsymbol{A}^{(k)}(t) \delta\left(\tau-\Delta \tau_{k}\right)
$$

where

$$
A^{(k)}(t)=\left\{a_{n m}^{(k)}(t)\right\}
$$

and

$$
a_{n m}^{(k)}(t)=\sum_{l=1}^{L} w_{\mathrm{TX}} w_{l m}^{(k)} c^{(k)} e^{j\left\{2 \pi f_{\mathrm{D} l} t+\frac{2 \pi}{\lambda}(n-1) d_{r} \cos \left(\theta_{l}-\theta_{0}\right)\right\}}
$$

The weight $w_{\mathrm{TX} \_l m}$ is the element of the TX connection matrix with row $l$ and column $m$ composed of $\mathrm{WH}$ codes to realize independent Rayleigh fading for each input port $m$. The coefficient $c^{(k)}$ is the amplitude of the $k$ th delayed wave, and the weight $w_{l}^{(k)}$ is the coefficient for probe antenna $l$ with delayed path $k$ to realize

$$
\begin{aligned}
& \text { Received } \boldsymbol{r}(t)=\boldsymbol{H}(t, \tau) \otimes \boldsymbol{s}(t)+\boldsymbol{n}(t) \quad(\otimes: \text { convolution integral })
\end{aligned}
$$

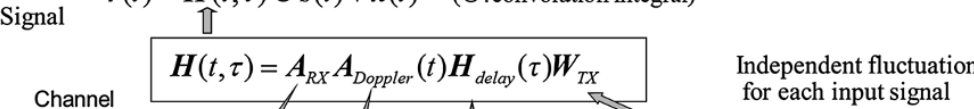

$$
\begin{aligned}
& \begin{array}{l}
\text { Characteristics } \\
\begin{array}{c}
\text { Doppler shift } \\
\text { Generation }
\end{array}
\end{array} \\
& \begin{array}{lllll}
A_{\text {Doppler }}(t)=\operatorname{diag}\left(e^{j 2 \pi f_{D 1} t}\right. & e^{j 2 \pi f_{D 2} t} & \cdots & \left.e^{j 2 \pi f_{D L} t}\right) f_{D l}=\frac{v}{\lambda} \cos \left(\theta_{l}+\Delta \theta_{l}\right) \\
\hline
\end{array} \\
& A_{R X}=\left(\begin{array}{llll}
v_{1} & v_{2} & \cdots & v_{L}
\end{array}\right) \\
& \boldsymbol{v}_{l}=\left(\begin{array}{llll}
v_{l 1} & v_{l 2} & \cdots & v_{l N}
\end{array}\right)^{\mathrm{T}} \\
& \text { Steering vector to the probe antenna } l
\end{aligned}
$$

Figure 4 Channel model of the proposed scheme. 
independent fluctuations of each delayed path. In the above equation, reception array is adopted as a linear equally spaced array $\left(d_{r}\right)$ in the direction of $\theta_{0}$ although the channel characteristics, $\boldsymbol{A}_{\mathrm{RX}}$, connecting the probe antenna array to the receiver antenna array as a DUT are more variable than we assumed here. The Doppler shift $f_{\mathrm{D} l}$ and direction of $\theta_{l}$ for probe antenna $l$ are discussed below.

Although the basic formulation has been given in Figure 4 [14], a detailed probe antenna arrangement rule was not given there. Therefore, we give the rule here. The Doppler shift matrix $\boldsymbol{A}_{\text {Doppler }}$ is the $L \times L$ diagonal matrix for the addition of the Doppler frequency shift to each probe antenna branch signal. In general, $\boldsymbol{A}_{\text {Doppler }}$ is automatically determined as a function of the probe antenna position in the direction of $\theta_{l}$ as well as the operational frequency $f$ and the assumed vehicular speed $v$. In order to avoid a symmetric arrangement of probe antenna positions in the assumed vehicular moving direction, which can cause the same Doppler shift on antenna pairs, we added a small angular offset to each probe antenna direction, as shown in Figure 5. The main considerations in the determination of $\theta_{l}$ are as follows:

1. We must avoid a symmetrical arrangement in the $x$-axis direction because the same Doppler shift frequency appears for the pair of directions. This reduces the variety of Doppler frequencies generated and works as if the number of probe antennas is smaller than the actual number $L$.

2. We must avoid a symmetrical arrangement in the $y$-axis direction because the arrangement cancels the imaginary part of the received signal for the pair of

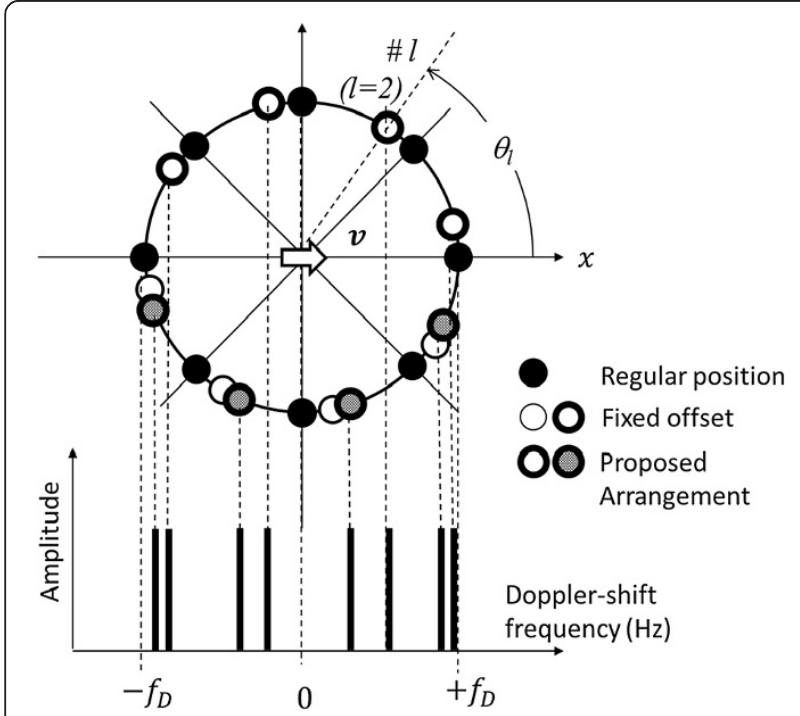

Figure 5 Doppler shift arrangement for each probe antenna. directions. This causes a deviation from Rayleigh distribution, as shown in Figure 6.

3. We must avoid a symmetrical arrangement about the center point for the same reason stated above.

The above Doppler shift setting problem is important only for the FE-2 system because each probe antenna in the FE-1 system radiates an independent Rayleigh fluctuating signal. In order to satisfy the above conditions, we adopted the following arrangement for $\boldsymbol{A}_{\text {Doppler: }}$

$$
\boldsymbol{A}_{\text {Doppler }}(t)=\frac{1}{\sqrt{L}} \operatorname{diag}\left(\begin{array}{llll}
e^{j 2 \pi f_{\mathrm{D} 1} t} & e^{j 2 \pi f_{\mathrm{D} 2} t} & \cdots & e^{j 2 \pi f_{\mathrm{D} L} t}
\end{array}\right)
$$

where

$$
\begin{aligned}
& f_{\mathrm{D} l}=\frac{v}{\lambda} \cos \left(\theta_{l}+\Delta \theta_{l}\right) \\
& \theta_{l}=\frac{2 \pi}{L}\left\{(l-1)+\frac{1}{4}\right\}
\end{aligned}
$$

and

$$
\Delta \theta_{l}=\left\{\begin{array}{rl}
0 & \text { for } l=1^{\sim} \frac{L}{2} \\
\frac{\pi}{4 L} & \text { for } l=\frac{L}{2}+1^{\sim} L
\end{array} .\right.
$$

The proposed arrangement is shown in Figure 5. The above Doppler shift setting rule appears to satisfy requirements 1 through 3 above. The validity of the assumption is examined in the next section.

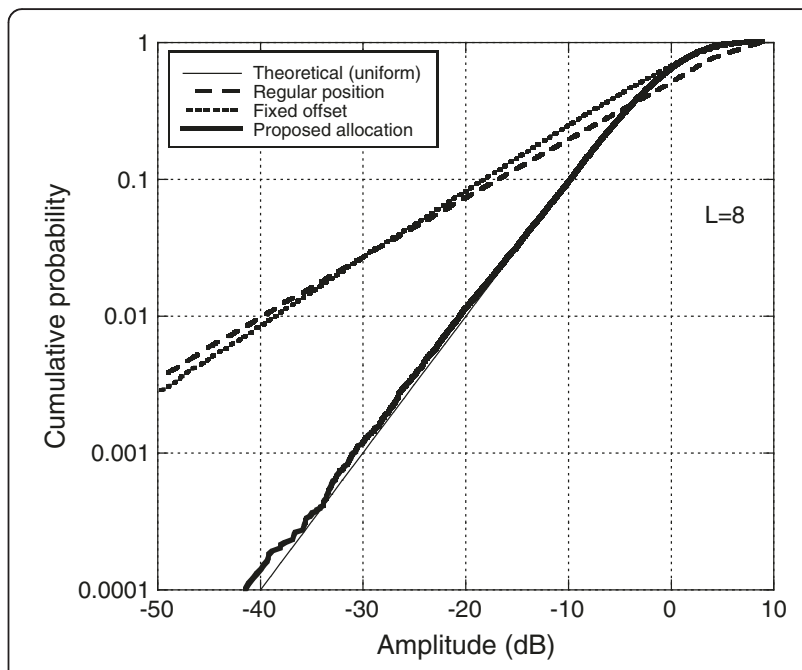

Figure $6 \mathrm{CDF}$ of amplitude distribution for three different Doppler shift arrangements. 


\section{Investigation of the generated channel performance}

\subsection{Single-input channel characteristics}

In this section, we consider the model having channel characteristics of $a_{11}^{(1)}(t)$ with $L=8$. We proposed the Doppler shift assignment shown in Figure 5. Figure 6 shows the cumulative distribution functions (CDFs) of the amplitude variations for three different Doppler shift arrangements, i.e., regular positions without any angular offsets, fixed offsets, and double offsets (proposed arrangement), with the theoretical Rayleigh distribution. The maximum Doppler shift $f_{\mathrm{D}}$ in this simulation is set to be $f_{\mathrm{D}} T_{\mathrm{s}}=0.01$, where $T_{\mathrm{s}}$ is the sampling period. Although the regular and fixed offset arrangements have a large discrepancy from the Rayleigh distribution, the proposed arrangement shows very good agreement.

Figure 7 shows the statistics of the time-varying amplitude for the proposed Doppler shift arrangement. Figure 7a shows the autocorrelation characteristics, and Figure $7 \mathrm{~b}$ shows the level crossing rate (LCR) statistics. Based on these figures, there is very good agreement with the theoretical values. Considering the results for the statistics, we adopt the proposed Doppler shift arrangement shown in Figure 5 for our analysis.

\subsection{Eigenvalue characteristics of generated MIMO channels}

Hereafter, we will give simulation results with parameters given in Table 1. The eigenvalues of the MIMO channel are important in evaluating the MIMO transmission performance characteristics, such as the bit error rate (BER) and the spectral efficiency in terms of channel capacity. In this section, we discuss the eigenvalue statistics of the correlation matrix $\boldsymbol{R}(t)$ defined by

$$
\boldsymbol{R}(t)=\boldsymbol{A}^{(1)}(t)\left\{\boldsymbol{A}^{(1)}(t)\right\}^{\mathrm{H}}
$$

where the superscript $\mathrm{H}$ shows the complex conjugate transpose of the matrix. From a practical viewpoint, it seems beneficial for a system to have the smallest possible number of probe antennas $L$. Since this requirement is counter to generating an accurate propagation environment, a quantitative evaluation is necessary in order to determine the minimum number of probe antennas $L$. Accordingly, we evaluate the eigenvalue statistics of an $N \times M$ MIMO system for $N=M=2,3$, and 4 as a parameter of $L$. The CDFs of the eigenvalues generated by the simulation and the corresponding independent and identically distributed (i.i.d.) Rayleigh cases are compared in Figure 8. As far as the eigenvalue characteristics are concerned, $L=8$ can realize an ideal environment for the $2 \times 2$ MIMO system, while $L=12$ or 16 can do the same for the $4 \times 4$ MIMO system.

For a more quantitative evaluation, we introduce the following measure. In the cumulative probability range of from 0.001 to 0.999 , when the maximum difference in decibels between the generated and theoretical eigenvalues is within $1 \mathrm{~dB}$, we define this case as 'excellent', that within $3 \mathrm{~dB}$ as 'good', that within $5 \mathrm{~dB}$ as 'fair', and that beyond $5 \mathrm{~dB}$ as 'poor', respectively. Table 2 shows this evaluation results for $M=N$ of 2,3 , and 4 with $L=8,12$, and 16. For comparison of the performance, similar simulations of FE-1 have also been carried out, and the obtained results are given in Table 2, which indicates that the performance of the FE-1 configuration is slightly superior to that of the FE-2. Even so, a very simple configuration of the antenna branch-controlled configuration (FE-2), which requires only $L$ Doppler shifters instead of the $M L K$ Rayleigh faders needed in the FE-1 system, has a significant advantage from a practical viewpoint.

In order to clarify the dependence of the performance on the number of probe antennas $L$ from a different

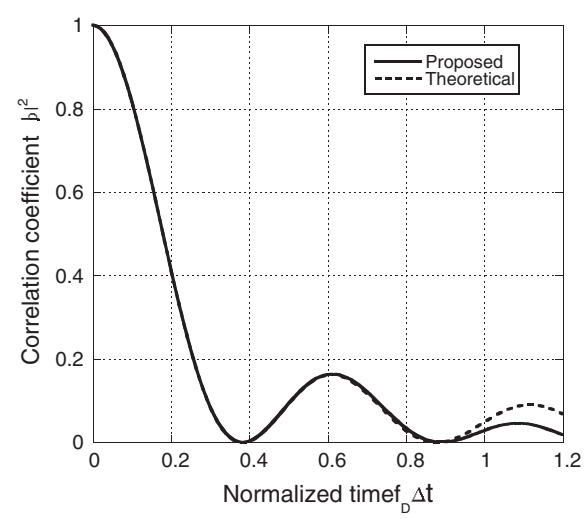

(a) Auto-correlation function

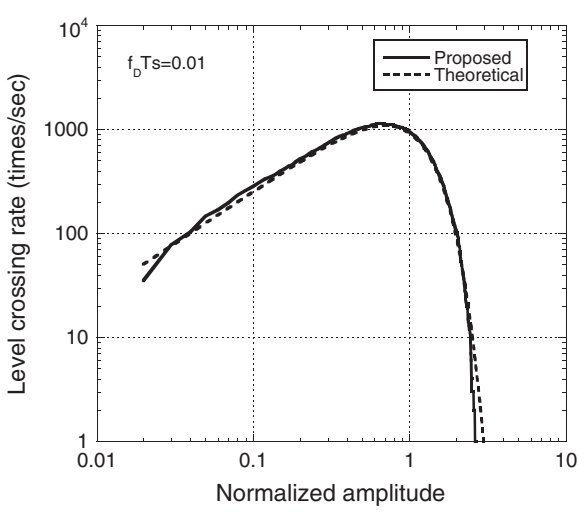

(b) Level-crossing rate (LCR) statistics

Figure 7 Statistics of temporal variations in amplitude. (a) Autocorrelation function. (b) LCR statistics. 
Table 1 Simulation parameters

\begin{tabular}{lc}
\hline Parameters & Value \\
\hline Input ports $(M)$ & $1 \sim 4$ \\
Probe antennas $(L)$ & $8,12,16$ \\
Receive antennas $(N)$ & $1 \sim 4$ \\
Delay paths $(K)$ & 1,6 \\
Normalized maximum Doppler shift $\left(f_{\mathrm{D}} T_{\mathrm{s}}\right)$ & 0.01 \\
Total sampling points & 100,000 \\
SNR for channel capacity calculation & $10 \mathrm{~dB}$ \\
\hline
\end{tabular}

point of view, we carried out the following error analysis for an $N \times N$ MIMO system:

$$
E=\frac{1}{N} \sum_{i=1}^{N} \frac{\left\langle\left|\lambda_{i}(p)-\lambda_{i, \text { theory }}(p)\right|\right\rangle}{\left\langle\lambda_{i, \text { theory }}(p)\right\rangle}
$$

where $\lambda_{i}(p)$ and $\lambda_{i \text {,theory }}(p)$ are the simulated and theoretical $i$ th eigenvalues corresponding to a cumulative probability of $p$. The above equation is used to estimate the
Table 2 Performance comparison of generated environments as viewed from the eigenvalue characteristics

\begin{tabular}{|c|c|c|c|c|c|c|}
\hline \multirow[t]{3}{*}{$L$} & \multicolumn{6}{|c|}{ Type } \\
\hline & \multicolumn{3}{|c|}{ FE-1 $(N \times M)$} & \multicolumn{3}{|c|}{ FE-2 $(N \times M)$} \\
\hline & $2 \times 2$ & $3 \times 3$ & $4 \times 4$ & $2 \times 2$ & $3 \times 3$ & $4 \times 4$ \\
\hline 8 & ○) & (๑) & $\Delta$ & (๑) & $\Delta$ & $x$ \\
\hline 12 & (อ) & (อ) & (อ) & O & O & O \\
\hline 16 & (-) & (-) & () & (๑) & (๑) & (อ) \\
\hline
\end{tabular}

normalized absolute deviation of eigenvalues in $\mathrm{CDF}$, where data for cumulative probabilities ranging from 0.001 to 0.999 were taken into consideration. The results are shown in Figure 9, where the $4 \times 4$ MIMO system with $L=8$ has a higher error for both FE-1 and FE-2. For rough estimation, $E=0.2$, which corresponds to a normalized eigenvalue of $1 \pm 0.2$, yields an error of approximately $1 \mathrm{~dB}$ so that the error region of $\mathrm{E}<0.2$ might be sufficiently accurate in practical application.

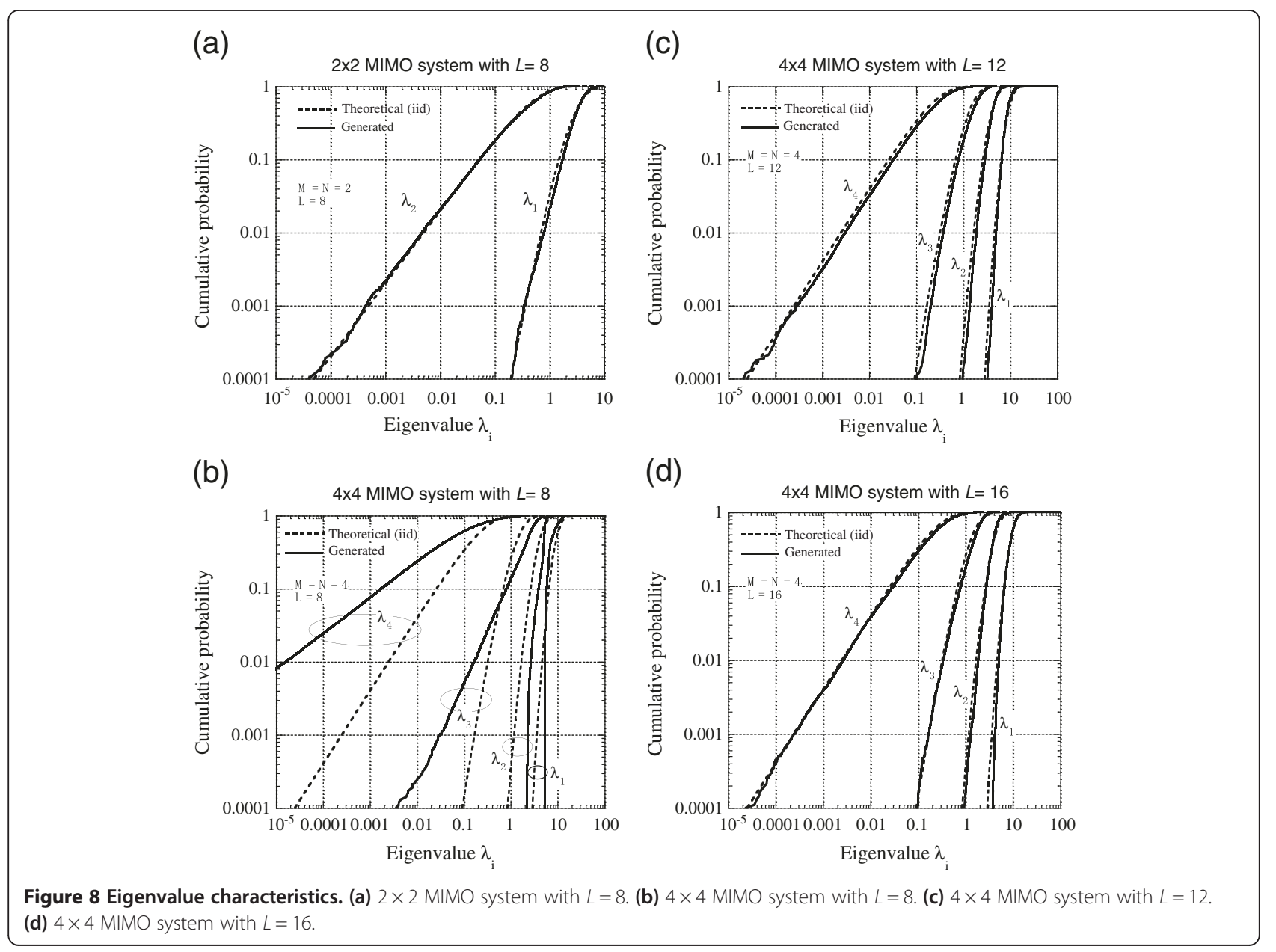




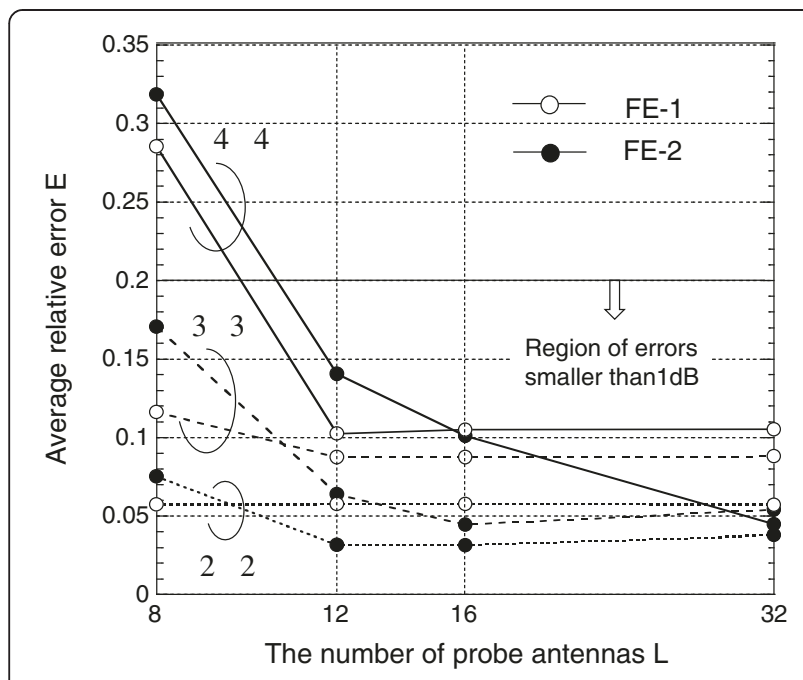

Figure 9 Normalized average error for an $N \times N$ MIMO system.

Thus, the $4 \times 4$ MIMO system with $L=8$ is insufficient from an eigenvalue analysis viewpoint.

Considering that the final performance evaluation measure is the digital transmission characteristic in terms of BER or spectral efficiency, i.e., often referred to as channel capacity, we evaluate the channel capacity performance (in bps/Hz), where equal powers are allocated to each input port. Figure 10 shows the results of the channel capacity comparison for a signal-to-noise ratio (SNR) of $10 \mathrm{~dB}$ for the $\mathrm{FE}-2$ cases shown in Table 2. In contrast to the large discrepancy of the eigenvalue characteristics shown in Figure $8 \mathrm{~b}$, the results for $L=8$ for the $4 \times 4$ MIMO system were in good

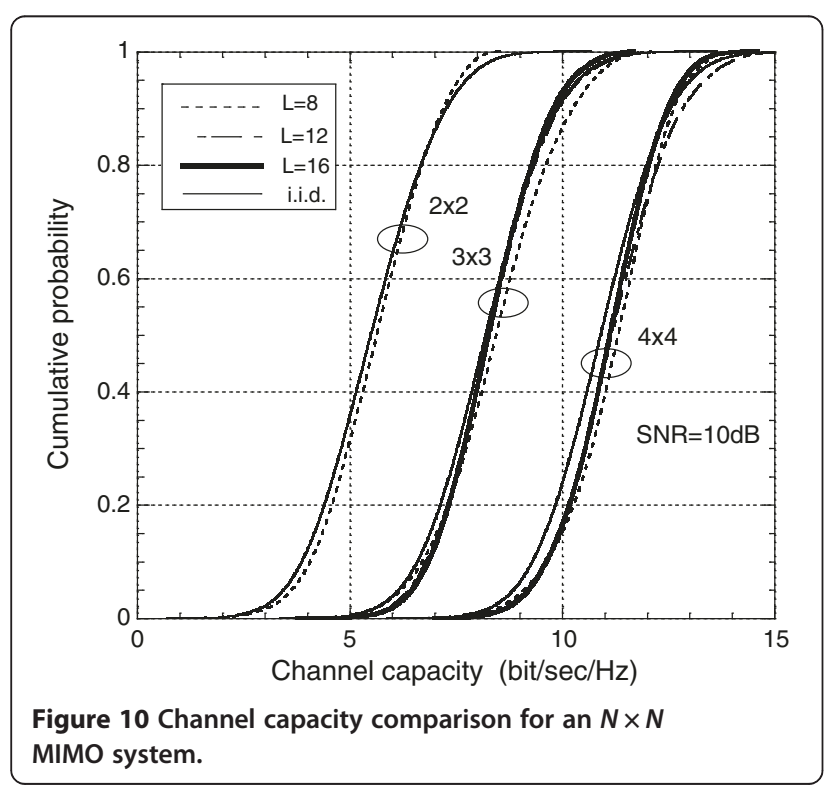

agreement. The average channel capacities for the $2 \times 2$ MIMO system with $L=8$ and i.i.d. are 5.63 and $5.55 \mathrm{bps} / \mathrm{Hz}$, respectively, whereas those for the $3 \times 3$ MIMO system with $L=8,12,16$, and i.i.d. are 8.47 , $8.35,8.31$, and $8.24 \mathrm{bps} / \mathrm{Hz}$, respectively. In addition, the average channel capacities for the $4 \times 4$ MIMO system with $L=8,12,16$, and i.i.d. are 11.23, 11.22, 11.11, and $10.93 \mathrm{bps} / \mathrm{Hz}$, respectively. The channel capacity evaluation indicates that the $L=8$ system can serve as a very good performance evaluation tool, even in the case of a $4 \times 4$ MIMO system.

In the case of eigenmode transmission with multistream transmission through each eigenmode path, the overall BER performance may depend on the individual eigenvalue characteristics. In such a case, $L=8$ for a $4 \times 4$ MIMO system is insufficient, as indicated in Figure 8b. However, the results of channel capacity evaluation shown in Figure 10 indicate that the performance depends primarily on the total sum of eigenvalues rather than on the individual eigenvalue characteristics. Figure 11 shows the CDF of the sum of four eigenvalues $\left(\lambda_{0}=\lambda_{1}+\lambda_{2}+\lambda_{3}+\lambda_{4}\right)$ in the $4 \times 4$ MIMO system for $L=8$, 12,16 , and 32 and the theoretical value under the i.i.d. fading condition. The figure shows that there are no significant differences even in the case of $L=8$. This is the reason why the channel capacity for the $4 \times 4$ MIMO system with $L=8$ shows good agreement with that for $L=12$ or 16 .

By adopting the $\mathrm{WH}$ code matrix for $\boldsymbol{W}_{\mathrm{TX}}$, i.i.d. fluctuations for each input port signal can be realized. In some, but not all, cases, an environment having spatial correlation on the transmitting side is required. In the case of $M=2$, with an arbitrary value of correlation

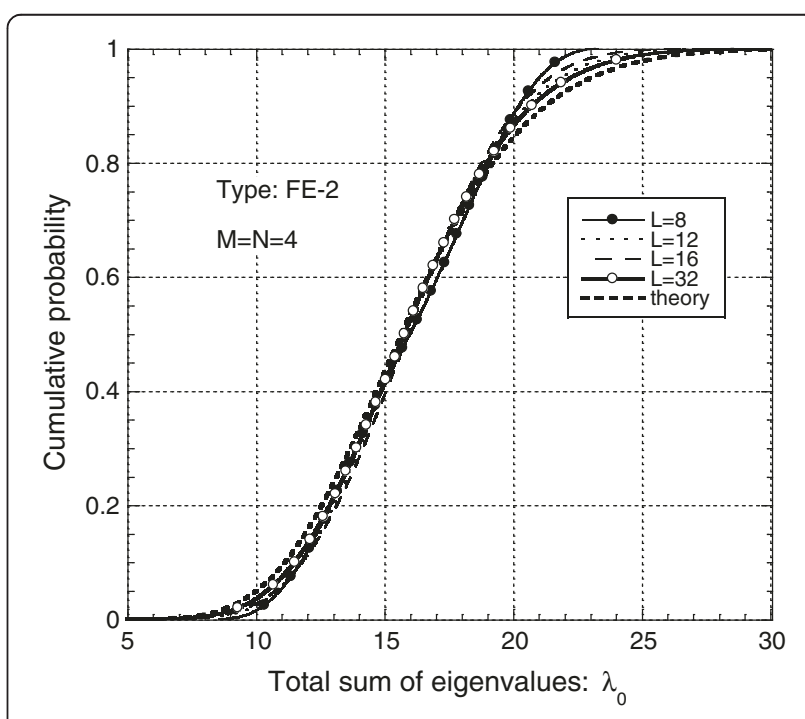

Figure $11 \mathrm{CDF}$ of the total sum of eigenvalues of a $4 \times 4$ MIMO system with different values of $L$. 
coefficient $\rho_{\mathrm{TX}}$ (real value assumed here) between two input ports, a candidate matrix $W_{\mathrm{TX}}$ is as follows:

$$
W_{\mathrm{TX}} \equiv\left(\begin{array}{cccccccc}
1 & 1 & 1 & 1 & 1 & 1 & 1 & 1 \\
b & b^{*} & b & b^{*} & b & b^{*} & b & b^{*}
\end{array}\right)^{\mathrm{T}}
$$

where

$$
\begin{aligned}
& b \equiv e^{j \phi / 2} \\
& \phi=2 \cos ^{-1}\left(\rho_{\mathrm{TX}}\right)
\end{aligned}
$$

For a $2 \times 2$ MIMO system, we carried out a Kronecker model simulation, and the results are shown in Figure 12, which shows that the expected amplitude variations having a $\mathrm{Tx}$-side spatial correlation are properly generated.

\subsection{Wideband characteristics}

Specific for wideband system is the fact that several waves with different delays can be resolved in the time domain. In computer simulations, it is easy to handle any number of delayed waves having any delay values. In the present paper, we consider a $2 \times 2$ MIMO channel with $L=8$ having six delayed waves. Figure 13 shows the CDF of each amplitude variation $\left|a_{11}^{(k)}\right|(k=1$ to 6$)$, where amplitude parameter $c^{(k)}$ is set at 1.0, 0.8, 0.6, 0.4, 0.2, and 0.1 for $k=1,2, \ldots, 6$, respectively. The CDFs of all of the generated delayed path amplitudes follow the

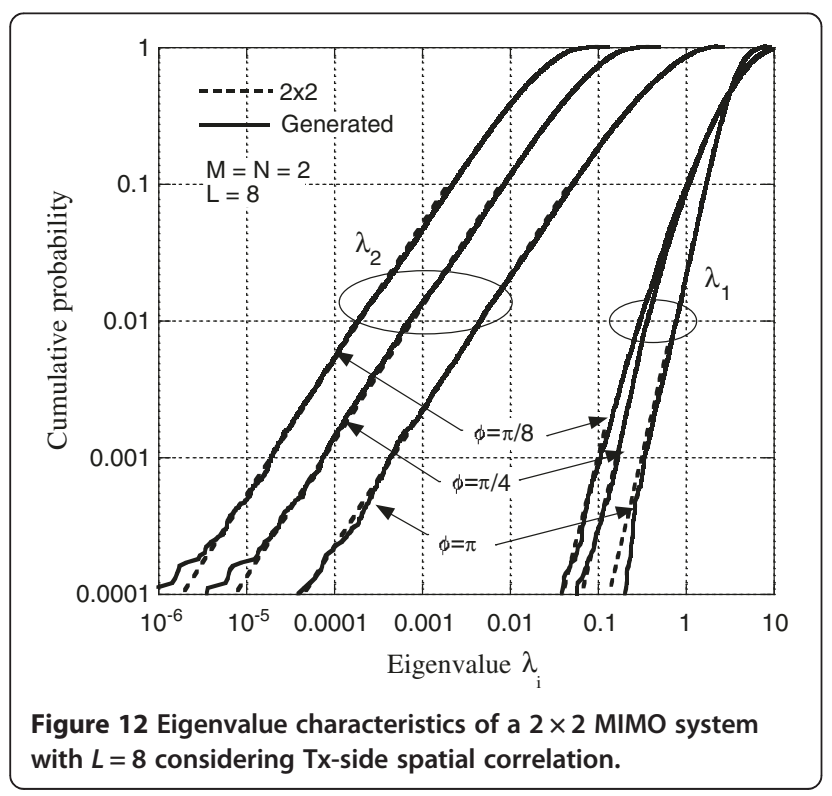

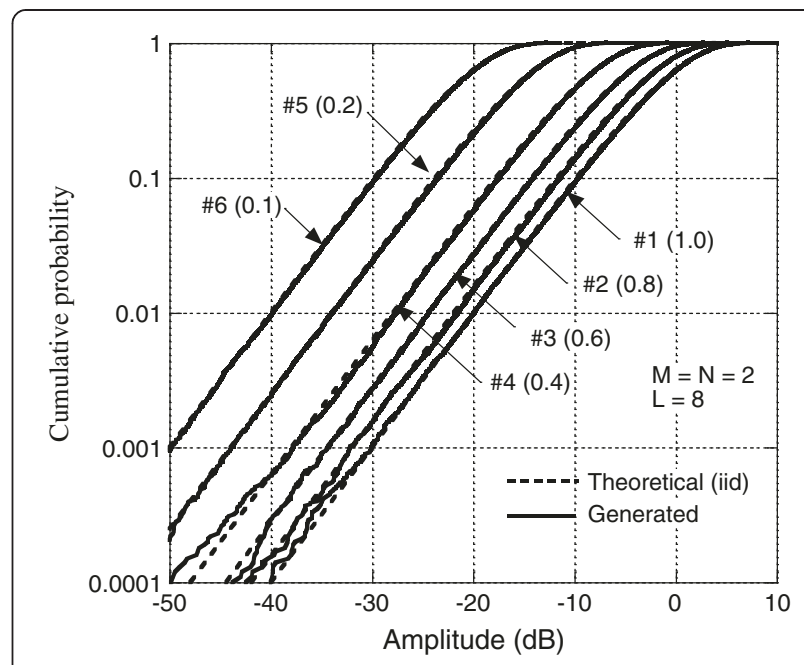

Figure $13 \mathrm{CDF}$ of amplitude variations for each delayed signal.

expected CDFs very accurately. The absolute values of the correlation coefficient $\rho_{1 m}{ }^{\left(k k^{\prime}\right)}$ where

$$
\rho_{m m^{\prime}}^{\left(k k^{\prime}\right)} \equiv \frac{\left\langle a_{1 m}^{(k) *} a_{1 m^{\prime}}^{\left(k^{\prime}\right)}\right\rangle}{\sqrt{\left\langle\left|a_{1 m}^{(k)}\right|^{2}\right\rangle\left\langle\left|a_{1 m^{\prime}}^{\left(k^{\prime}\right)}\right|^{2}\right\rangle}}
$$

are given in Table 3. The values in the table, except for those in italics, are approximately 0 or 1 because we can give four orthogonal WH code sets of $L=8$ for the weight factor $w_{l}^{(k)}$ in Equation 2c. Accordingly, almostperfect independent Rayleigh fluctuations are generated

Table 3 Correlation characteristics among generated delayed waves

\begin{tabular}{lcccccc}
\hline & $a_{11}^{(1)}$ & $a_{11}^{(2)}$ & $a_{11}^{(3)}$ & $a_{11}^{(4)}$ & $a_{11}^{(5)}$ & $a_{11}^{(6)}$ \\
\hline$a_{11}^{(1)}$ & 1 & 0.0069 & 0.0095 & 0.0063 & 0.0087 & 0.4966 \\
$a_{11}^{(2)}$ & 0.0069 & 1 & 0.0037 & 0.0124 & 0.0055 & 0.0053 \\
$a_{11}^{(3)}$ & 0.0095 & 0.0037 & 1 & 0.0071 & 0.0027 & 0.0031 \\
$a_{11}^{(4)}$ & 0.0063 & 0.0124 & 0.0071 & 1 & 0.0013 & 0.4969 \\
$a_{11}^{(5)}$ & 0.0087 & 0.0055 & 0.0027 & 0.0013 & 1 & 0.004 \\
$a_{11}^{(6)}$ & 0.4966 & 0.0053 & 0.0031 & 0.4969 & 0.004 & 1 \\
$a_{12}^{(1)}$ & 0.0048 & 0.0029 & 0.006 & 0.0053 & 0.4986 & 0.0083 \\
$a_{12}^{(2)}$ & 0.0041 & 0.0062 & 0.0012 & 0.0015 & 0.504 & 0.4983 \\
$a_{12}^{(3)}$ & 0.0055 & 0.0009 & 0.0076 & 0.0023 & 0.5047 & 0.5033 \\
$a_{12}^{(4)}$ & 0.0029 & 0.0092 & 0.0023 & 0.0054 & 0.4972 & 0.0108 \\
$a_{12}^{(5)}$ & 0.5023 & 0.4986 & 0.498 & 0.5061 & 0.0021 & 0.0071 \\
$a_{12}^{(6)}$ & 0.008 & 0.5007 & 0.4958 & 0.0079 & 0.0073 & 0.0039 \\
\hline
\end{tabular}

The values in the table, except for those in italics, are approximately 0 or 1 because we can give four orthogonal WH code sets of $L=8$ for the weight factor $w_{l}^{(k)}$ in Equation $2 c$. 
among delayed waves corresponding to $k=1$ to 4 . For delayed waves corresponding to $k=5$ and 6 , the correlation coefficients are not always 0 because we assigned weighting factors for the delayed waves using random numbers of 1 or -1 due to the lack of an orthogonal code set for $L=8$ [14]. When selecting the weighting factors for $k=5$ and $k=6$, we excluded random code sets having a correlation of more than 0.5 . That is the reason why the obtained correlation values are not more than 0.5 . All of the delayed waves, including those for $k=5$ and 6 , can therefore be generated as expected.

\section{Conclusions}

We evaluated a simplified configuration for OTA test systems. The key element of the proposed model is the adoption of an antenna branch-controlled configuration (FE-2) for generating multipath delayed waves. We introduced an orthogonal code weighting for generated delayed waves to realize independent amplitude fluctuations among all of the generated delayed paths with multiple input ports, and the performance of the generated channel was evaluated.

Based on the results of the analysis, we reached the following conclusions:

1. By assigning the appropriate Doppler shift to each probe antenna branch, very accurate Rayleigh fluctuations for both amplitude distribution and temporal variation statistics can be generated using eight-probe antennas.

2. From the viewpoint of eigenvalue analysis in generating the MIMO propagation channel, at least 8 -probe antennas are necessary for evaluating the $2 \times 2$ MIMO system, whereas 12 - or 16 -probe antennas are necessary for the $3 \times 3$ and $4 \times 4$ MIMO systems.

3. From the viewpoint of channel capacity analysis, eight-probe antennas are sufficient, even in the case of $4 \times 4$ MIMO system measurement.

4. By introducing orthogonal code set weighting, almost-independent Rayleigh fluctuations of delayed paths for different input ports are realized with a very simple configuration based on the antenna branch-controlled scheme.

One of the primary practical advantages of the proposed scheme is the realization of a flexible MIMOOTA testing system in a very simplified configuration without the loss of necessary functions or a reduction in performance for the purpose of measurement. Due to the way the fading functions are configured in a cascade, an implementation of the scheme into an FPGA circuit is promising from a practical viewpoint. One disadvantage of the proposed scheme is the difficulty involved in the flexible control of a Tx connection matrix excepting a case of $M=2$, as compared to a path-controlled configuration (FE-1).

The necessary number of probe antennas in an FE OTA system increases with increasing complexity of the radiation pattern of the DUT or the angular power profile of the multipath environment, such as a multicluster environment. Moreover, a dual polarization system requires twice the number of probe antennas. For practical applications, typical standard channel models described in Section 6.2 in [5] should be evaluated using the proposed model. Since the proposed channel model has the capability to realize such standard channel models by setting the parameter values appropriately, we can show calculation examples. However, it seems very difficult to present the evaluation results with a systematic comparison. Since very careful evaluations are necessary, these evaluations are left for future studies.

\section{Competing interests}

The authors declare that they have no competing interests.

Received: 17 August 2013 Accepted: 25 November 2013

Published: 17 December 2013

\section{References}

1. JP Kermoal, L Schumacher, KI Pedersen, PE Mogensen, F Frederiksen, A stochastic MIMO radio channel model with experimental validation. IEEE J Sel Areas Commun 20(6), 1211-1226 (2002)

2. AF Molish, A generic model for MIMO wireless propagation channels in macro- and microcells. IEEE Trans Signal Proc 52(1), 61-71 (2004)

3. MA Jensen, JW Wallace, A review of antennas and propagation for MIMO wireless communications. IEEE Trans Antennas Propagat 52(11), 2810-2824 (2004)

4. W Weichselberger, M Herdin, H Ozcelik, E Bonek, A stochastic MIMO channel model with joint correlation of both link ends. IEEE Trans Wireless Commun 5(1), (2006)

5. 3GPP, Measurement of radiated performance for MIMO and multi-antenna reception for HSPA and LTE terminal. Tech Rep (2012). TR37.976 v11.0.0. http://www.quintillion.co.jp/3GPP/Specs/37976-b00.pdf

6. H Arai, Measurement of Mobile Antenna Systems (Artech House, London, 2001)

7. PS Kildal, K Rosengren, Correlation and capacity of MIMO systems and mutual coupling radiation efficiency, and diversity gain of their antennas: simulation and measurements in a reverberation chamber. IEEE Commun Mag 42(12), 104-111 (2004)

8. P Kyosti, JP Nuutinen, T Jaemsa, MIMO OTA test concept with experimental and simulated verification, in 2010 Proceedings of the Fourth European Conference on Antennas \& Propagation, Barcelona, April 2010 (IEEE, Piscataway, 2010)

9. H Iwai, K Sakaguchi, T Sakata, A Yamamoto, Proposal of spatial fading emulator dedicated for performance evaluation of handset antennas. IEICE Trans Commun (Japanese Ed. J91-B(9), 960-971 (2008)

10. T Sakata, A Yamamoto, K Ogawa, J Takada, MIMO channel capacity measurement in the presence of spatial clusters using a fading emulator, in 2009 20th IEEE International Symposium on Personal, Indoor and Mobile Radio Communications, Tokyo, September 2009 (IEEE, Piscataway, 2009), pp. 97-101

11. C Park, J Takada, K Sakaguchi, T Ohira, Spatial fading emulator for base station using cavity-excited circular array based on ESPAR, in 2004 60th IEEE Vehicular Technology Conference, Los Angeles, September 2004 (IEEE, Piscataway, 2004), pp. 256-260

12. L Rudant, C Delaveaued, M AbouElAnouar, Synthesizing realistic environments in an anechoic chamber, in 3rd European Conference on Antennas and Propagation, Berlin, March 2009 (IEEE, Piscataway, 2009), pp. $221-225$ 
13. A Scannavini, LJ Foged, MAE Anouar, N Gross, J Estrada, OTA throughput measurements by using spatial fading emulation technique, in Fourth European Conference on Antennas and Propagation, Barcelona, April 2010 (IEEE, Piscataway, 2010), pp. 1-5

14. A Kosako, M Shinozawa, Y Karasawa, A simplified configuration of fading emulator system for MIMO-OTA testing. IEICE Trans Commun (Japanese Ed.) J95-B(2), 275-284 (2012)

15. A Kosako, M Shinozawa, Y Karasawa, Simplified configuration of fading emulator system for MIMO-OTA testing, in 2011 2nd International Conference on Wireless Communication, Vehicular Technology, Information Theory and Aerospace \& Electromagnetic Systems Technology, Chennai, February-March 2011 (IEEE, Piscataway, 2011)

16. Y Karasawa, Y Gunawan, S Pasisingi, K Nakada, A Kosako, Development of a MIMO-OTA system with simplified configuration. J Korean Inst Electromagn Eng Sci 12(1), 77-84 (2012)

17. P Dent, GE Bottomley, T Croft, Jakes fading model revisited. Electron Lett 29(13), 1162-1163 (1993)

doi:10.1186/1687-1499-2013-285

Cite this article as: Pasisingi et al.: Performance evaluation of a MIMO channel model for simplified OTA test systems. EURASIP Journal on

Wireless Communications and Networking 2013 2013:285.

Submit your manuscript to a SpringerOpen ${ }^{\circ}$ journal and benefit from:

- Convenient online submission

- Rigorous peer review

- Immediate publication on acceptance

- Open access: articles freely available online

- High visibility within the field

- Retaining the copyright to your article

Submit your next manuscript at $\gg$ springeropen.com 